\title{
Life Style-Related Diseases of the Digestive System: Endocrine Disruptors Stimulate Lipid Accumulation in Target Cells Related to Metabolic Syndrome
}

\author{
Koichiro Wada ${ }^{1, *}$, Hirotada Sakamoto ${ }^{2}$, Kenji Nishikawa ${ }^{2}$, Satoru Sakuma ${ }^{2}$, Atsushi Nakajima ${ }^{3}$, \\ Yohko Fujimoto ${ }^{2}$, and Yoshinori Kamisaki ${ }^{1}$ \\ ${ }^{1}$ Department of Pharmacology, Graduate School of Dentistry, Osaka University, \\ 1-8 Yamadaoka, Suita, Osaka, 565-0871, Japan \\ ${ }^{2}$ Department of Physiological Chemistry, Osaka University of Pharmaceutical Sciences, \\ 4-20-1, Nasahara, Takatsuki, Osaka 569-1094, Japan \\ ${ }^{3}$ Gastroenterology Division, Yokohama University School of Medicine, \\ 3-9 Fuku-ura, Kanazawa-ku, Yokohama 236-0004, Japan
}

Received July 10, 2007; Accepted September 10, 2007

\begin{abstract}
Many reports indicated that endocrine disruptors (EDs) affect several hormonal functions in various living things. Here, we show the effect of EDs on lipid accumulation in target cells involved in the onset of metabolic syndrome. Treatment with nonylphenol and bisphenol A, typical EDs, stimulated the accumulation of triacylglycerol in differentiated adipocytes from 3T3-L1, preadipocytes, in time- and concentration-dependent manners. Up-regulation of gene expressions involved in lipid metabolism and metabolic syndrome were observed in adipocytes treated with EDs. Similarly, stimulatory effects of EDs were also observed on the human hepatoma cell line HuH-7. These observations indicate that exposure to EDs stimulates the lipid accumulation in target cells involved in the metabolic syndrome and may cause the dysfunction of those cells, resulting in induction of metabolic syndrome.
\end{abstract}

Keywords: life style-related disease, endocrine disruptor, metabolic syndrome, adipocyte, hepatocyte

\section{Introduction}

Diabetes mellitus, hyperlipidemia, hypertension, and atherosclerosis have recently been defined as typical life style-related diseases. The common background of these diseases is obesity. Recently, the incidence of obesity and associated metabolic syndrome has been dramatically increased. Although high caloric foods like those found in a typical Western style diet are believed to represent the root of such a dramatic increase, there may be other possible causes. It is plausible and provocative to relate the recent increased incidence of metabolic syndrome to a dramatic increase in the use of industrial chemicals over the past 50 years. Namely,

*Corresponding author. kwada@dent.osaka-u.ac.jp

Published online in J-STAGE: October 6, 2007

doi: 10.1254 /jphs.FM0070034 exogenously obesity-inducible substances, "Environmental Obesogens", are now considered as the one of the potential causes of obesity and associated metabolic syndrome $(1,2)$. Especially, environmentally existing xenobiotic chemicals, such as endocrine disruptors (EDs), would be probable candidates.

EDs are a great concern throughout the world because they have adverse effects on human beings. However, most of previous reports about the adverse effects of EDs have been concerned with the reproductive system $(3-6)$. We previously reported the inhibitory effect of EDs on neural stem cell proliferation and differentiation, indicating the possibility that EDs may influence various physiological systems as well as the reproductive system (7). Therefore, investigations for other potential adverse effects by EDs are required. In fact, high concentrations of EDs, such as nonylphenol or bisphenol A, exist in the environment. Nonylphenol is used in surfactants or 
A<smiles>CCc1ccc(O)cc1</smiles>

4-Nonylphenol

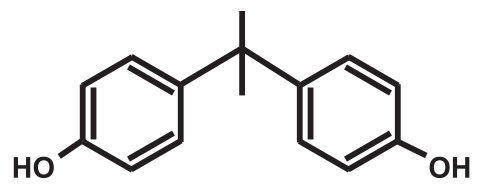

Bisphenol A
B

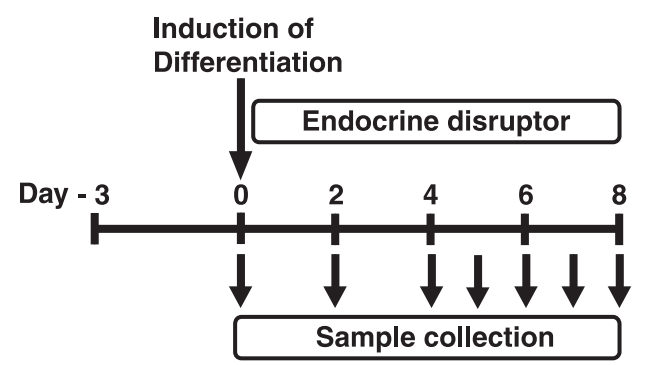

Fig. 1. Structures of endocrine disruptors and schematic protocol. A: Structures of endocrine disruptors (EDs) used in the present study (4-nonylphenol and bisphenol A). B: Schematic protocol of adipocyte differentiation. The 3T3L-1 preadipocytes were confluent during the culture for 3 days, and then induction of differentiation was started by the addition of insulin, dexamethazone, 3-isobutyl-1methylxanthine, or rosiglitazone, a PPAR $\gamma$ agonist. Endocrine disruptors $(0.1-10 \mu \mathrm{M})$ were administered to the culture system at the start of induction (Day 0). Samples were collected at the points indicated.

plastic and petroleum processing, and bisphenol A is used in polycarbonate plastics or dental resin-based composites.

In this review, we summarize the effect of EDs on lipid accumulation in target cells, such as adipocytes and hepatocytes, related to the metabolic syndrome that induce diabetes, fatty liver, hyperlipidemia, and atherosclerosis. We selected nonylphenol and bisphenol A, two well-known EDs, , for the investigation because they are widely used and comparatively high concentrations of them were detected in the environment (Fig. 1A).

\section{Nonylphenol and bisphenol A stimulate accumula- tion of triacylglycerol in adipocytes}

The adipocytes in visceral fat are considered to be the most important cells involved in the onset of metabolic syndrome $(8-10)$. We, therefore, applied the EDs nonylphenol and bisphenol A to 3T3-L1 preadipocytes to see their effects on the differentiation to mature adipocytes (Fig. 1B, Ref. 11). Treatment with nonylphenol and bisphenol A each significantly stimulated the accumulation of triacylglycerol, a marker of lipid accumulation, in mature adipocytes differentiated from 3T3-L1 preadipocytes (Fig. 2: A and B); the response to each ED was time- and concentration-dependent (data not shown). Interestingly, increase in cell size was observed in the nonylphenol-treated adipocytes, but not in the bisphenol A-treated ones. These results indicate the possibility that both nonylphenol and bisphenol A can promote the differentiation of preadipocytes to mature adipocytes.

\section{Nonylphenol and bisphenol A stimulate accumula- tion of triacylglycerol in hepatocytes}

Hepatocytes in the liver are also considered to play important roles in metabolic syndrome because the liver controls the metabolism of various substances including lipids (9). Excessive lipid accumulation in hepatocytes leads to liver dysfunction, resulting in further aggravation of the systemic condition. Therefore, we applied nonylphenol and bisphenol A to $\mathrm{HuH}-7$ cells, a human hepatocellular carcinoma cell line, to investigate their effects on lipid accumulation (12). Treatment with nonylphenol and bisphenol A caused time- and dosedependent, significant increases in glycerol accumulation (data not shown). These results clearly indicate that nonylphenol and bisphenol A have the ability to stimulate the lipid accumulation not only in adipocytes but also in hepatocytes (Fig. 4).

\section{Major alteration of gene expression in adipocytes treated with EDs}

To investigate the alterations of genes treated with EDs, we performed exhaustive analysis of genes using a DNA microarray. Up-regulated gene expressions involved in lipid metabolism, adipocyte differentiation, and inflammation were observed by the treatment with nonylphenol (Fig. 3). Namely, the up-regulation of hormone-sensitive lipase, phospholipase $\mathrm{A}_{2}$, and phospholipase $\mathrm{C}$ that are involved in lipid metabolism were observed by the treatment with nonylphenol. In addition, up-regulation of $\mathrm{CD} 36$, a receptor for oxidized- or acetylated-LDL, and TNF- $\alpha$, an important adipocytokine to induce insulin resistance, were also observed $(8-10)$. In contrast, up-regulation of the adiponectin gene was much smaller than those of phospholipase and TNF- $\alpha$. Similar results were also observed when cells were treated with bisphenol A (data not shown). These results clearly indicate that EDs accelerate the differentiation of preadipocytes to mature adipocytes, 
A

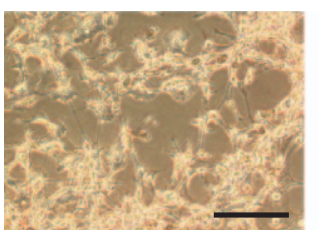

Vehicle

B
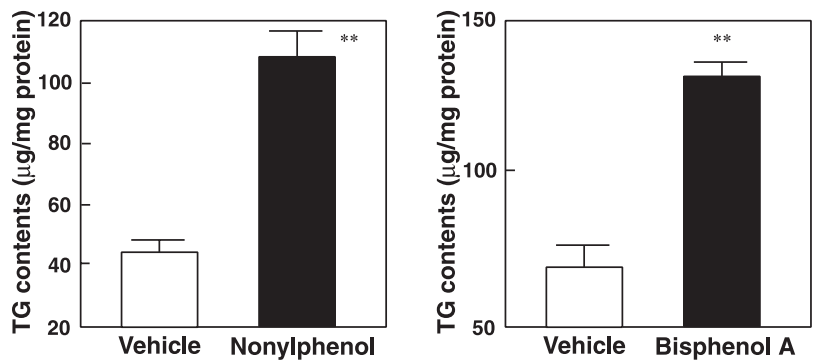

Fig. 2. Effects of EDs on lipid accumulation. A: Typical photographs of differentiated adipocytes from 3T3L-1 preadipocytes treated with nonylphenol $(3 \mu \mathrm{M})$ or vehicle for 5 days. Cells were stained by the Oil Red $\mathrm{O}$ method to visualize lipid accumulation. Scale bar is $200 \mu \mathrm{m}$. B: Effects of nonylphenol and bisphenol A on lipid accumulation in differentiated adipocytes. The 3T3-L1 cells were treated with nonylphenol $(10 \mu \mathrm{M}$, left panel) or bisphenol A $(10 \mu \mathrm{M}$, right panel) for 5-6 days. Then, accumulation of triacylglycerol (TG), a marker of lipid accumulation, in mature adipocytes was measured and expressed as TG contents. Each column represents the mean \pm S.E.M. from $5-8$ independent experiments. ${ }^{* *} P<0.01$ vs vehicle control.
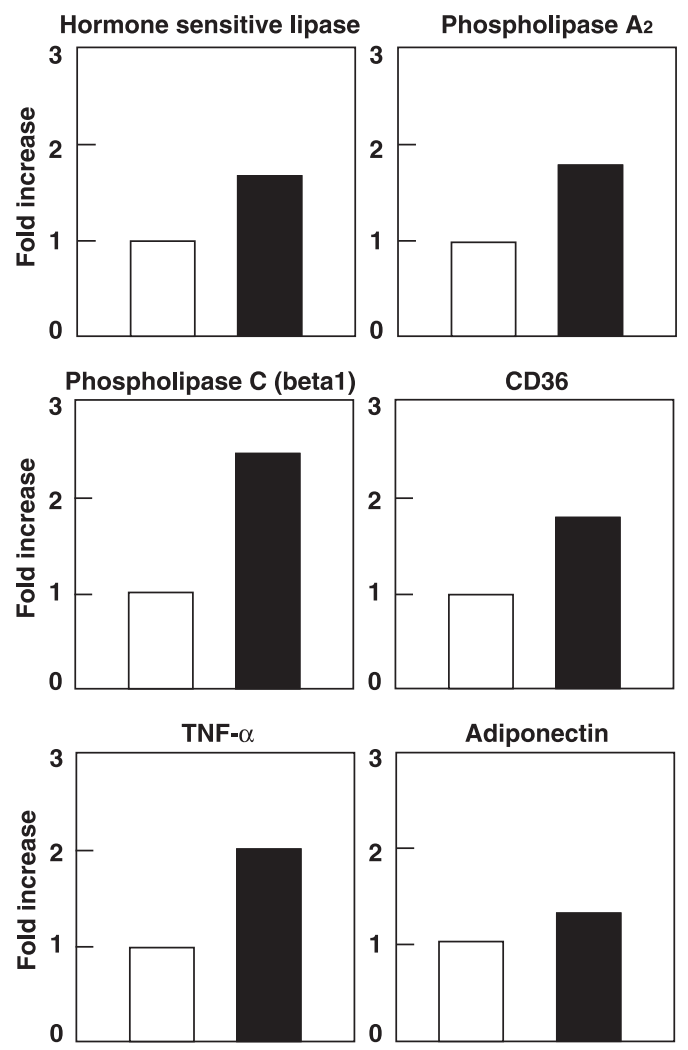

Fig. 3. Major alterations of gene expression in preadipocytes treated with nonylphenol on differentiated adipocytes. The 3T3L-1 preadipocytes were treated with nonylphenol $(10 \mu \mathrm{M})$ for 4 days, and then total RNA was prepared and applied to DNA microarray analysis. Various genes were altered, and the major altered genes related to lipid metabolism and metabolic syndrome are shown. Fold increase of gene expression by nonylphenol treatment (closed column) compared to vehicle treatment (open column) is shown.

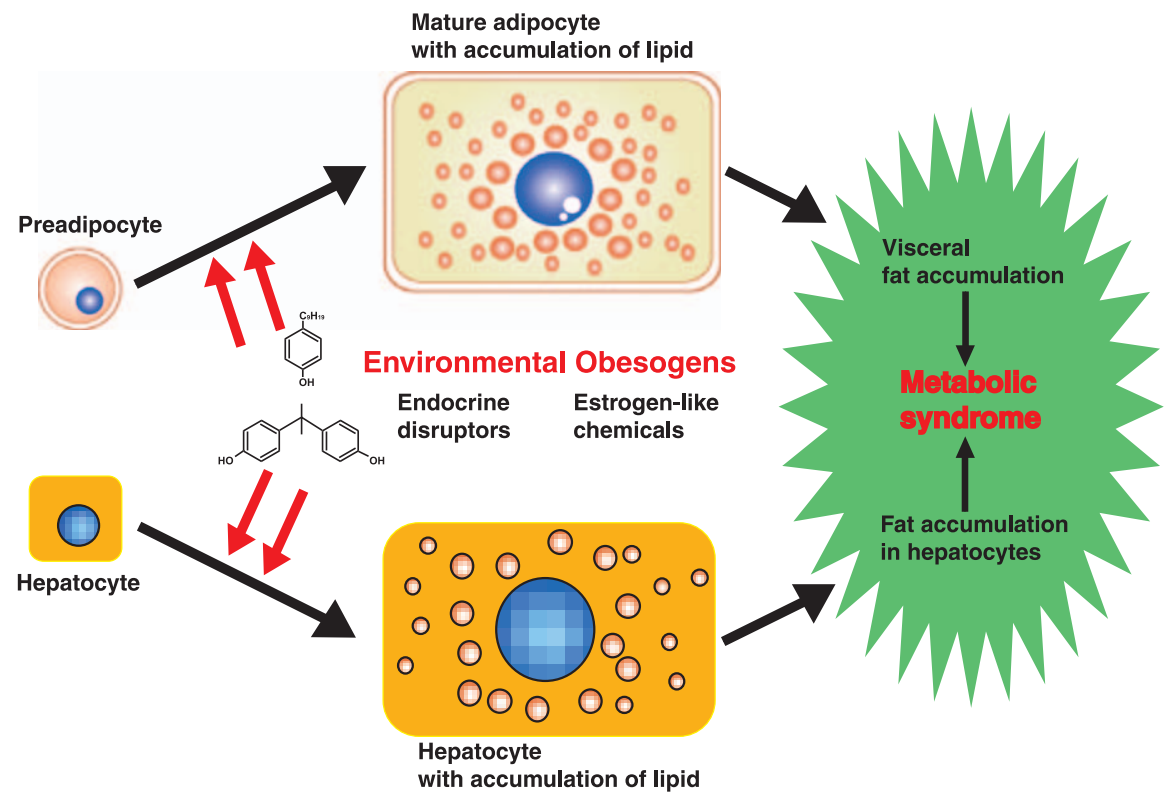

Fig. 4. Schematic illustration of possible involvement of EDs in lipid accumulation in adipocytes and hepatocytes. 
resulting in the accumulation of lipids in the cell body. In addition, the direction of differentiation is toward enlargement of cell size, indicating large adipocytes rather than small adipocytes. Because adiponectin is mainly released from small adipocytes $(8-10)$, these results suggest that the acceleration of adipocyte differentiation by EDs aggravate the condition of metabolic syndrome (Fig. 4).

\section{Concluding remarks}

So far, it is not fully understood whether environmental chemicals such as EDs promote the onset of metabolic syndrome. However, several recent reports, including our present observations, indicate that EDs have the potential to stimulate the lipid accumulation in target cells, such as adipocytes and hepatocytes, related to obesity and associated metabolic syndrome. These observations suggest that EDs are possible environmental obesogens, exogenously obesity-inducible xenobiotic chemicals. It has also been advocated that organotins are potent environmental obesogens because tributyltin promotes adipocyte differentiation via the peroxisome proliferator-activated receptor gamma $(\operatorname{PPAR} \gamma)$ pathway $(2,13-15)$. Thus, the mechanism of adipocyte differentiation by organotins is comparatively well understood. In contrast, the mechanisms of the other EDs have not been fully investigated. Several reports indicated that most of the EDs such as nonylphenol and bisphenol A bind to estrogen receptors, resulting in their actions on the reproductive system $(2-6)$. However, it is doubtful whether the effect on adipocyte differentiation by nonylphenol and bisphenol A may be mediated solely through the estrogen receptor signaling pathway because these observations, including ours, are in conflict with the general conclusions that estrogens are potentially anti-adipogenic $(2,16,17)$. Therefore, other mechanisms could be involved in the adipocyte differentiation stimulated by nonylphenol and bisphenol $\mathrm{A}$, and further investigations are required to clarify this point.

Another important issue is whether the target cells can actually be environmentally exposed to concentrations such as those used in previous reports and our study. Most of previous reports used nonylphenol or bisphenol $\mathrm{A}$ in the range of $1-30 \mu \mathrm{M}$ in their in vitro studies. In contrast, we also observed the stimulation of lipid accumulation in the concentration range of $30 \mathrm{nM}$ to $10 \mu \mathrm{M}$. It may be possible that in normal circumstances, the level of nonylphenol or bisphenol A in contaminated water or foods is still lower than the effective concentration on lipid accumulation in the present study. However, nonylphenol has been reported to appear in the aquatic environment, particularly in sediment, and can reach up to $3000 \mathrm{ppb}$ in rivers and lakes (18). In addition, it has been reported that lipidsoluble environmental pollutants, including nonylphenol, accumulate to high concentrations in animal and human tissues (19). Furthermore, we detected the accumulation of nonylphenol and bisphenol $\mathrm{A}$ in liver, visceral fat, pancreas, and other target organs in mice orally administered with these compounds (unpublished data). Therefore, low-level chronic or high-level acute exposure to EDs might increase their blood and tissue concentrations in animals or humans to reach the levels high enough to elicit the effects on the target cells to stimulate lipid accumulation.

In conclusion, exposure to EDs stimulates the lipid accumulation in target cells involved in metabolic syndrome and may cause the dysfunction of those cells, resulting in induction of metabolic syndrome.

\section{References}

1 Mokdad AH, Ford ES, Bowman BA, Dietz WH, Vinicor F, Bales VS, et al. Prevalence of obesity, diabetes, and obesityrelated health risk factors. JAMA. 2003;289:76-79.

2 Grun F, Blumbeg B. Environment obesogens: Organotins and endocrine disruption via nuclear receptor signaling. Endocrinol. 2006;147 Suppl:S50-S55.

3 Kelce WR, Gray LE, Wilson EM. Antiandrogens as environmental endocrine disruptors. Reprod Fertil Dev. 1998;10:105111.

4 Sonnenscein C, Soto AM. An updated review of environmental estrogen and androgen mimics and antagonists. J Steroid Biochem Mol Biol. 1998;65:143-150.

5 Ohtani H, Miura I, Ichikawa Y. Effects of dibutyl phthalate as an environmental endocrine disruptor on gonadal sex differentiation of genetic males of the frog Rana rugosa. Environ Health Prospect. 2000;108:1189-1193.

6 Cupp AS, Skinner MK. Actions of the endocrine disruptor methoxychlor and its estrogenic metabolite on in vitro embryonic rat seminiferous cord formation and perinatal testis growth. Reprod Toxicol. 2001;15:317-326.

7 Kudo C, Wada K, Masuda T, Yonemura T, Shibuya A, Fujimoto $\mathrm{Y}$, et al. Nonylphenol induces the death of neural stem cells due to activation of the caspase cascade and regulation of the cell cycle. J Neurochem. 2004;88:1416-1423.

8 Hiragum A, Sato M, Mitsui H. Preadipocyte differentiation in vitro: identification of a highly active adipogenic agent. J Cell Physiol. 1988;134:124-130.

9 Kadowaki T, Hara K, Yamauchi T, Terauchi Y, Tobe K, Nagai R. Molecular mechanism of insulin resistance and obesity. Exp Biol Med. 2003;228:1111-1117.

10 Fantuzzi G. Adipose tissue, adipokines, and inflammation. J Allergy Clin Immunol. 2005;115:911-919.

11 Katayama K, Wada K, Miyoshi H, Ohashi K, Tachibana M, Furuki R, et al. RNA interfering approach for clarifying the PPARgamma pathway using lentiviral vector expressing short hairpin RNA. FEBS Lett. 2004;560:178-182. 
12 Heller T, Saito S, Auerbach J, Williams T, Moreen TR, Jazwinski A, et al. An in vitro model of hepatitis $\mathrm{C}$ virion production. Proc Natl Acad Sci U S A. 2005;102:2579-2583.

13 Golub M, Doherty J. Triphenyltin as a potential human endocrine disruptor. J Toxicol Environ Health B Crit Rev. 2004;7: 281-295.

14 Saitoh M, Yanase T, Morinaga H, Tanabe M, Mu YM, Nishi Y, et al. Tributyltin or triphenyltin inhibits aromatase activity in the human granulosa-like tumor cell line KGN. Biochem Biophys Res Commun. 2001;289:198-204.

15 Kanayama T, Kobayashi N, Mamiya S, Nakanishi T, Nishikawa J. Organotin compounds promote adipocyte differentiation as agonists of the peroxisome proliferator-activated receptor $\gamma$ /retinoid X receptor pathway. Mol Pharmacol. 2005;67:766774.

16 Murata Y, Robertson KM, Jones ME, Simpson ER. Effect of estrogen deficiency in the male: the ArKO mouse model. Mol Cell Endocrinol. 2002;193:7-12.

17 Heine PA, Taylor JA, Iwamoto GA, Lubahn DB, Cooke PS. Increased adipose tissue in male and female estrogen receptor- $\alpha$ knockmice. Proc Natl Acad Sci U S A. 2000;97:12729-12734.

18 Naylor CG, Mieure JP, Adams WJ, Weeks JA, Castaldi FJ, Ogle $\mathrm{LD}$, et al. Alkylphenol ethoxylates in the environment. J Am Oil Chem Soc. 1992;69:695-703.

19 Tapiero H, Ba GN, Tew KD. Estrogens and environmental estrogens. Biomed Pharmacother. 2002;56:36-44. 